

RNA sequencing reveals sexually dimorphic gene expression before gonadal differentiation in chicken and allows comprehensive annotation of the W-chromosome

Ayers et al. 


\title{
RNA sequencing reveals sexually dimorphic gene expression before gonadal differentiation in chicken and allows comprehensive annotation of the W-chromosome
}

Katie L Ayers ${ }^{1,2,3+}$, Nadia M Davidson ${ }^{1 \dagger}$, Diana Demiyah ${ }^{4}$, Kelly N Roeszler ${ }^{1}$, Frank Grützner ${ }^{5}$, Andrew H Sinclair ${ }^{1,2,6}$, Alicia Oshlack ${ }^{1 *}$ and Craig A Smith ${ }^{1,2,6^{*}}$

\begin{abstract}
Background: Birds have a ZZ male: ZW female sex chromosome system and while the Z-linked DMRT1 gene is necessary for testis development, the exact mechanism of sex determination in birds remains unsolved. This is partly due to the poor annotation of the $\mathrm{W}$ chromosome, which is speculated to carry a female determinant. Few genes have been mapped to the $W$ and little is known of their expression.

Results: We used RNA-seq to produce a comprehensive profile of gene expression in chicken blastoderms and embryonic gonads prior to sexual differentiation. We found robust sexually dimorphic gene expression in both tissues pre-dating gonadogenesis, including sex-linked and autosomal genes. This supports the hypothesis that sexual differentiation at the molecular level is at least partly cell autonomous in birds. Different sets of genes were sexually dimorphic in the two tissues, indicating that molecular sexual differentiation is tissue specific. Further analyses allowed the assembly of full-length transcripts for $26 \mathrm{~W}$ chromosome genes, providing a view of the $\mathrm{W}$ transcriptome in embryonic tissues. This is the first extensive analysis of $\mathrm{W}$-linked genes and their expression profiles in early avian embryos.

Conclusion: Sexual differentiation at the molecular level is established in chicken early in embryogenesis, before gonadal sex differentiation. We find that the $\mathrm{W}$ chromosome is more transcriptionally active than previously thought, expand the number of known genes to 26 and present complete coding sequences for these $W$ genes. This includes two novel W-linked sequences and three small RNAs reassigned to the W from the Un_Random chromosome.
\end{abstract}

Keywords: Sex determination, Embryonic chicken gonad, W chromosome, Avian sex, RNA-seq

\section{Background}

Sex determination in mammals and birds occurs at fertilization, with the differential inheritance of sex chromosomes [1]. Mammals have an XX/XY sex chromosome system, characterised by male heterogamety $(\mathrm{XY})$, while birds have a ZZ/ZW system and female heterogamety $(\mathrm{ZW})$. In eutherian mammals, almost all genes on the $\mathrm{Y}$

\footnotetext{
* Correspondence: alicia.oshlack@mcri.edu.au; craig.smith@mcri.edu.au † Contributed equally

'Murdoch Childrens Research Institute, Royal Childrens Hospital, Flemington Road, Parkville 3054 Melbourne, VIC, Australia

Full list of author information is available at the end of the article
}

chromosome are associated with male development and fitness, namely the testis-determining $S R Y$ gene and genes required for spermatogenesis [2]. The $\mathrm{Z}$ and $\mathrm{W}$ sex chromosomes of birds evolved independently of the eutherian $\mathrm{X}$ and $\mathrm{Y}$, and hence birds lack the $S R Y$ gene [3]. Like the mammalian $\mathrm{X}$ chromosome, the avian $\mathrm{Z}$ chromosome is large and gene-rich, while the $\mathrm{W}$, like the mammalian $\mathrm{Y}$, is typically small and largely heterochromatic. The $82 \mathrm{Mb}$ chicken $\mathrm{Z}$ chromosome harbours over 1,000 genes and is highly conserved among avians $[4,5]$, while the $\mathrm{W}$ has far fewer genes and appears to have undergone independent degradation across the

\section{() BiolMed Central}


various avian groups [6,7]. The exact roles of $\mathrm{Z}$ and $\mathrm{W}$ genes in avian sex determination remain to be fully resolved. Sex determination may involve a dominantacting female determinant carried on the female-specific $\mathrm{W}$ chromosome, or a $\mathrm{Z}$ dosage-based mechanism could prevail [8]. Consistent with the latter, the Z-linked $D M R T 1$ and HEMOGEN genes are involved in testis development in the chicken embryo $[9,10]$. However, a potential role for the W sex chromosome in avian sex determination cannot yet be excluded.

Attempts to address the potential role of the W chromosome in avian sex determination have been hampered by the poor understanding of this chromosome and its gene content. The chicken W chromosome has an estimated size of approximately $55 \mathrm{Mb}$, most of which (at least 70\%) comprises repetitive elements of the XhoI, EcoR 1 and $S s p 1$ classes [11,12]. In addition, long arrays of interstitial telomeric sequences have been described on the $\mathrm{W}$. The exact number of genes on the $\mathrm{W}$ is obscure. The chicken genome, from a female Red Jungle fowl, was sequenced in 2004 [13] but assembly and annotation of the $\mathrm{W}$ has remained incomplete, and it is estimated that only $4 \%$ of the chicken W has been mapped [14]. This amounts to approximately $1.2 \mathrm{Mb}$ of assembled sequence on the $\mathrm{W}$ in the most recent release of the Chicken genome (Gallus_gallus-4.0; November 2011) [15]. Until recently, around 12 bona fide genes had previously been verified as W-linked, including CHD1W, ATP5A1W, HINTW, UBAP2W, NIPBL, hnRNPW, KCMF, SMAD2, SPIN, MIER3, ZFR and ZNF532 [6,12,16-20] (chicken genome release 67, Ensembl) [21]. In a study of W chromosome gene expression in different breeds of chickens, Moghadam and colleagues recently increased the number of putative W genes to 21, with several genes suggested to have multiple copies [22]. However, expression data for these and other $\mathrm{W}$ genes at critical time points during embryonic sexual differentiation are lacking.

It has recently been proposed that avian sexual differentiation is at least partially cell autonomous. Cell autonomous sexual differentiation would involve purely intrinsic genetic factors, independent of any extrinsic signalling. In contrast, the long-held dogma is that vertebrate sexual differentiation is non-cell autonomous, that is, it does not rely purely on intrinsic factors in the cell and involves some degree of extrinsic signalling. (Sex hormones secreted from the gonads to induce female or male sexual dimorphisms, for example.) Zhao and colleagues (2010) used data from naturally occurring gynandomorphic chickens (birds that are half male and half female) and cross-sex embryonic tissue transplantations to show that sexual differentiation may be cell autonomous, at least partly independent of hormones [23]. This study would predict molecular sexual differentiation (that is, sexually dimorphic gene expression) prior to morphological sexual differentiation (that is, development of testes or ovaries and other sex dimorphisms) (reviewed in [24]). However, large-scale data in support of this hypothesis in avians have not previously been reported in any detail.

This study uses RNA sequencing to examine the degree of cell autonomous sexual differentiation in birds and to characterise the $\mathrm{W}$ transcriptome in chicken embryos. Comprehensive analysis of gene expression in early embryos reveals significant sexually dimorphic gene expression well prior to gonadal sex differentiation, involving both the sex chromosomes and autosomes. These findings support the idea that avian sexual differentiation is at least partly cell autonomous at the molecular level [23]. Different sets of genes showed sexual dimorphism in chicken blastoderms versus gonads, indicating that distinctive molecular pathways underlying sexual differentiation operate in different tissues. In addition, this analysis has allowed the full characterisation of gene expression from the enigmatic W (female) sex chromosome. Full characterisation of these $\mathrm{W}$ transcripts allows definitive comparisons with their $\mathrm{Z}$ homologues, shedding light on avian sex determination.

\section{Results}

\section{Expression profiling of blastoderms and embryonic day} 4.5 gonads reveals at least partial cell autonomous molecular sexual differentiation in chicken

Deep transcriptome sequencing was used to profile gene expression at two developmental time points in males and females; 12-h blastoderms (Hamburger and Hamilton stage 1) and day 4.5 embryonic gonads (stage 26) [25]. The rationale for using these times points was our focus on sex determination. Blastoderms represent the earliest accessible post-laying developmental stage, prior to primitive streak formation and gastrulation. This stage was chosen to specifically address the question of cell autonomous molecular sex differentiation pre-dating morphological differentiation. The second tissue, embryonic gonads at day 4.5 , represents the time when the gonads are still morphologically identical in each sex ('bipotential').

Sequenced read-pairs were mapped to the chicken genome, (galGal3), using the TopHat 1.3.1 software [26]. The overlap of read-pairs with Ensembl genes was then counted. Differential expression analysis was undertaken by testing the female counts against male counts at both time-points using edgeR [27], with a false discovery rate $($ FDR $)<0.05$. Genes known to be expressed sexually dimorphically in E4.5 gonads served as positive controls. For example, $D M R T 1$ and $A M H$ are known to be male upregulated by approximately two-fold in E4.5 gonads, and FOXL2 is expressed only in female gonads at E5.0. Meanwhile, both Aromatase and SOX9 are expressed after E4.5, and were expected to be non-dimorphic in our datasets [28]. These patterns were confirmed in the 
RNA-seq (see Additional file 1, Figure S1), validating the sequencing results.

Our annotation-based differential expression analysis [29] revealed hundreds of genes differentially expressed between males and females in both tissues (362 in blastoderms and 357 in the gonads) (Figure 1A, and Additional file 2 and 3). This indicated robust sexually dimorphic gene expression pre-dating gonadal development, and supports the notion of cell autonomous sexual differentiation at the molecular level in chicken. In the blastoderm, most of the genes upregulated in males were Z-linked (85\%), with a smaller but significant proportion annotated to autosomes (12\%) (Figure 1B). This indicated that the $\mathrm{Z}$ chromosome is not fully dosage compensated, with the mean expression of Z-linked genes 1.6-fold higher in males compared with females (see Additional file 1 Figure S2), as reported previously [30-32]. Meanwhile genes upregulated in females were annotated to the $\mathrm{W}$ chromosome (38\%), autosomes (39\%), or to the Un_random chromosome (21\%) (Figure 1B). The latter represents a virtual chromosome of un-assembled and un-localised chicken sequence. A similar trend was observed in the E4.5 gonads (Figure 1B). Notably, a very small number $\mathrm{Z}$ linked sequences were female-biased, in both tissues (Figure 1B). These derived from the $M H M$ locus (Male Hypermethylated), a curious sequence that has previously been reported to be female specific and hypothesised to play a role in localised dosage compensation (upregulation of some neighbouring $\mathrm{Z}$ genes in females) [33,34].

Some genes involved in sexual differentiation of the gonads begin to be dimorphically expressed between the sexes only in the gonads (for example, FOXL2). These genes therefore showed a different pattern of sexual dimorphism from blastoderm to gonad; that is, the female: male ratio was significantly different between tissues. To identify additional genes showing this difference in sexual dimorphism, we used a robust statistical test for the difference in female: male fold change between tissues (see Additional file 1 and 4). Of all of the 43 W-linked Ensembl sequences we detected, none of these genes showed a different pattern of sexual dimorphism (Figure 1C). For Zgenes, of which 262 were differentially expressed in at least one tissue, only three genes (1\%) showed a significantly different pattern of sexual dimorphism between tissues (Figure 1C). This included two MHM locus genes that show a large increase in female expression in the gonads, and the Endothelial Tyrosine Kinase (TEK) gene that was more dimorphic in blastoderm (ENSGALG0 0000018479, ENSGALG00000023324, ENSGALG0000 0001840). However, 40 autosomal genes (25\%) and 22 Un_random genes (45\%) showed a different pattern of sexual dimorphism between tissues (Figure 1C). These data indicate that sex-specific molecular pathways that manifest in the blastoderms differ to those in gonads, primarily due to difference in autosomal gene expression. Given that sex chromosome genes did not generally deviate in their female: male ratio between the two tissues, it is interestingly to speculate how these genes can then activate different downstream genes in blastoderms and gonads. In the case of DMRT1, a known testis determinant that is differentially expressed with a similar ratio in both tissues, we found that the average expression level ((male+female)/2) of this gene dramatically increased from blastoderm to gonad. We therefore tested all genes for significant differential expression between tissues, regardless of sex (see Additional files 1 and 4). Indeed, a number of sexually dimorphically expressed $\mathrm{W}$ and $\mathrm{Z}$ genes also showed differential expression between the tissues, that is, W-genes 23 (53\%), Z-genes - 194 (74\%) (Figure 1D and Additional file 4). Those genes upregulated in the gonads (Figure 1D and Additional file 4) are therefore interesting candidate gonadal sexual differentiation genes. Altogether, the data indicate that the relative expression levels of dimorphic sex-linked genes could explain their ability to regulate different downstream genes in different tissues.

To shed light on the molecular pathways that might underlie cell-autonomous sexual differentiation, we first screened the datasets for genes implicated in gonadal sex differentiation. A list was compiled of 117 genes previously linked tovertebrategonadogenesis (see Additional file 5). The set of genes differentially expressed between male and female blastoderms was significantly enriched with these gonadogenesis genes $(P=0.0098$, Fisher's exact test), mostly due to non-compensated Z-linked genes (for example, 17ßHSDB4 (ENSGAL00000002187), DMRT1 (ENSGAL00000010160) and CFC1B (ENSGA L00000012623)). Only one differentially expressed autosomal 'implicated gonadal gene' was found differentially expressed in this tissue (VNN1, ENSGALG00000013992) (Additional file 2). No genes previously proven to have a role in gonadal sex differentiation were found among the W-linked sequences. Taken together, these data indicate that sex chromosome genes do not activate known sexual differentiation pathways in blastoderms of either sex.

In contrast to the blastoderms, the gonads showed a different set of differentially expressed autosomal genes, many of which have a known link to gonadal sex differentiation, such as FOXL2, Anti-Müllerian Hormone (AMH), INHA (Inhibin-A) and HSP70 (ENSGALG00000011715). This indicates that the sex chromosomes have initiated developmental programs specifically associated with gonadal sex differentiation at embryonic day 4.5 (stage 25), 1 to 2 days prior to the onset of gonadal sexual differentiation (stages 29-30).

These findings show that different sexually dimorphic molecular pathways are engaged by the sex chromosomes in the two different tissues examined here. However, most of the differentially expressed genes detected 


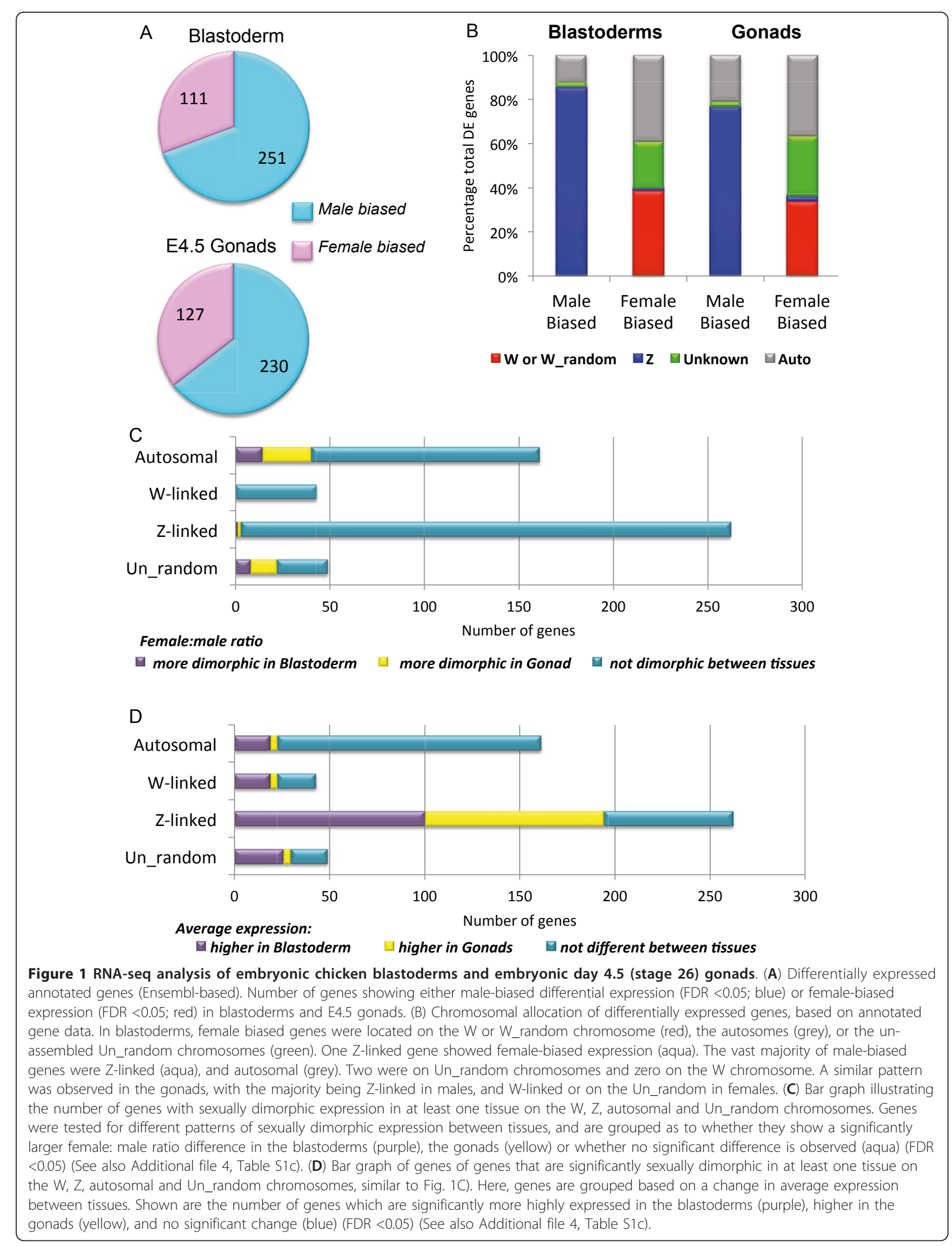


in blastoderms and gonads had no previous connection to sexual differentiation. To characterise potential pathways activated in female versus male tissues, we assessed gene ontology of all genes that were exclusively differentially expressed in only blastoderms or gonads, using the DAVID programs [35]. The top three clusters of GO terms for each group are shown in (Additional file 1, Figure S10). Given the low number of genes, very few GO terms showed significant enrichment when we corrected for multiple testing (Benjamini), however numerous genes involved in cell stress and DNA damage repair showed sex differential expression in the blastoderm. Various members of the hepatic fibrosis pathway were also differentially expressed ( $A 2 M$ and Col3A1 upregulated in females, and IL1R2 and EGF in males). Sex-specific gene expression in the liver has been previously described, involving $>1,000$ genes that affect a wide range of biological processes [36]. For genes specifically differentially expressed in the gonads, the top GO terms included neuroactive ligand-receptor interaction $(P$ value $=0.0081)$. These genes include the GABA receptor alpha 4 and two glutamate receptors. Among the list of autosomal genes expressed in the day 4.5 gonads in a sexually dimorphic fashion were several transcription factors not previously linked to sex per se (four upregulated in females and six in males) (Additional file 2). These data reveal the engagement of different molecular pathways at the two tissues assayed.

As mentioned previously, Z- and W-linked genes generally showed similar dimorphic expression in both blastoderms and gonads (Figure 1C), while many autosomal genes were only differentially expressed in one tissue (Figure 1C). In addition, a significant proportion of genes annotated to the Un_Random chromosome showed a stable dimorphic expression profile (Figure 1C). These unassigned sequences may in fact map to sex chromosomes, especially the poorly annotated W chromosome. Evidence for this conclusion was supported by their sex expression ratios, which were similar to those of the sex chromosome genes (Additional files 2 and 3). As the chicken W sex chromosome and its potential role in avian sex determination is currently poorly understood, we exploited the RNA-seq data to definitively annotate these sequences and to address W-linked gene expression.

\section{Annotation of the W chromosome}

Given the robust W-linked gene expression in both blastoderms to E4.5 gonads, we considered that this chromosome might play an important role in sex determination and cell autonomous molecular sex differentiation. However, the chicken W sex chromosome is currently incompletely annotated and its sequence is only partially assembled. In light of the challenges associated with sequencing the $\mathrm{W}$ chromosome, we investigated the $\mathrm{W}$ transcriptome and its potential role in avian sex determination in more detail. Two approaches were used to construct full-length open reading frames for the W transcriptome, genome-guided and genome independent (de novo) assembly.

Potential mis-annotated $\mathrm{W}$ genes were initially identified by their female specific expression (Ensembl) (see Methods and Materials). However, a significant fraction of reads also mapped outside annotated genes. Most notably, $62 \%$ of reads that mapped to the Un_random chromosome were not in annotated genes (Additional file 1, Figure S3). We hypothesised that some of these sequences were unannotated W-linked contigs. In order to identify unannotated genes, we extended our analysis of the RNAseq data using a genome guided transcript assembly procedure, Cufflinks [37]. The Cufflinks analysis was performed by mapping to the chicken genome (Galgal4), and a set of chicken transcripts was created by running Cufflinks 1.3.0 on the mapped reads (see Additional file 1). A significant proportion of expressed W genes identified through the Cufflinks analysis encoded retroviral elements with at least one open reading frame that showed homology to a retroviral protein (with an e-value $<0.001$, using BLAST). Together with pseudogenes, these sequences were filtered out of subsequent analysis (see Methods and Materials and Supplementary Methods in Additional file1).

This analysis allowed the compilation of $26 \mathrm{~W}$-linked genes (Table 1). Most of these genes have previously been suggested or confirmed to be W-linked [22], but we identified two novel W-linked sequences, TXN-like and SUB1-W. In addition, two W-linked genes, RPL17$\mathrm{W}$ and $H N R P K-W$, lie on the Un_random chromosome as described in [22] and confirmed by us. Located within the intronic region of these genes are two annotated small nucleolar RNAs (SNORD58-W-1 and SNORD58-W 2) and one microRNA $(m I R-7 b-W)$ that have not been documented to be W-linked. Give the location of the host gene and the presence of a gametologue on the $\mathrm{Z}$ chromosome, these genes should be reassigned to the $\mathrm{W}$ chromosome.

During both the Ensembl and Cufflinks analyses, it was noted that in at least 12 cases, multiple identified transcripts encoded the same putative protein (Suppl. Table 3). While in some cases, such as HINT-W and FAF, this was due to multiple copies of the gene in the genome, in many cases there was a single copy of the gene, but it had been split across non-contiguous or gapped regions of the genome. Genome-guided assemblies such as Cufflinks are limited by the quality of the genome and transcripts cannot be assembled across segments of the genome which are not correctly scaffolded or which contain gaps in the assembled sequence. This is particularly true for the Un_random and W_random chromosomes, which contain unassembled 
Table 1 Annotation and expression of chicken $\mathbf{W}$ chromosome genes.

\begin{tabular}{|c|c|c|c|c|c|c|c|}
\hline \multirow[t]{2}{*}{ Gene } & \multirow[t]{2}{*}{ Description } & \multirow[t]{2}{*}{ Genomic Location } & \multicolumn{2}{|c|}{ Expression (FPKM) Female } & \multicolumn{2}{|c|}{ \% ID with paralog } & \multirow[t]{2}{*}{$\mathrm{dN} / \mathrm{dS}$} \\
\hline & & & Blastoderms & E4.5 Gonads & DNA & Protein & \\
\hline ATP5A1-W & ATP synthase subunit alpha & $\begin{array}{l}\text { ChrW } \\
\text { W_random }\end{array}$ & 101 & 155 & 89.3 & 95.4 & 0.280 \\
\hline BTF3-W & Transcription factor BTF3-like & Autosome & 86.3 & 141 & 90.9 & 94.4 & 0.160 \\
\hline C18ORF25-W & Uncharacterized protein C18ORF25 & $\begin{array}{l}\text { ChrW } \\
\text { W_random ChrUn }\end{array}$ & 0.874 & 3.93 & 90.3 & 83.7 & 0.434 \\
\hline CHD1-W & Chromodomain helicase DNA binding protein & $\begin{array}{l}\text { ChrW } \\
\text { W_random autosomal }\end{array}$ & 6.81 & 9.35 & 86.8 & 88.1 & 0.126 \\
\hline$\overline{F A F}$ & Female Associated Factor & $\begin{array}{l}\text { ChrW } \\
\text { W_random }\end{array}$ & 15 & 21.2 & & & \\
\hline GOLPH3-W & Golgi phosphoprotein 3-like & ChrUn & 2.82 & 24.9 & 92.7 & 95.6 & 0.080 \\
\hline HINT-W & Histidine triad nucleotide binding protein & $\begin{array}{l}\text { ChrW } \\
\text { W_random }\end{array}$ & 1780 & 1170 & 41.0 & 48.5 & 0.608 \\
\hline HNRPK-W & Heterogeneous nuclear ribonucleoprotein $\mathrm{K}$ & ChrUn & 103 & 100 & 94.2 & 99.3 & 0.040 \\
\hline KCMF1-W & E3 ubiquitin-protein ligase KCMF1-like & $\begin{array}{l}\text { ChrW } \\
\text { W_random }\end{array}$ & 10.1 & 21.6 & 92.0 & 93.2 & 0.181 \\
\hline MIER3-W & Mesoderm induction early response 3 & ChrUn & 7.42 & 19.9 & 92.9 & 92.5 & 0.265 \\
\hline NEDD4-like-W & Neural precursor cell expressed developmentally downregulated 4-like & Chrun & 0.048 & 1.92 & 82.4 & 83.0 & 0.209 \\
\hline NIPBL-W/SCC2-W & Nipped B/sister chromatid cohesion 2 & $\begin{array}{l}\text { ChrW } \\
\text { W_random }\end{array}$ & 7.78 & 7.39 & 89.9 & 90.0 & 0.148 \\
\hline RASA1-W & Ras GTPase-activating protein 1-like & $\begin{array}{l}\text { ChrW } \\
\text { W_random ChrUn }\end{array}$ & 2.77 & 10.5 & 81.4 & 89.4 & 0.132 \\
\hline RPL17-W & Ribosomal protein 17 & ChrUn & 368 & 332 & 96.7 & 100.0 & 0.000 \\
\hline SMAD2-W & Mothers against decapentaplegic homolog 2-like & $\begin{array}{l}\text { ChrW } \\
\text { W_random }\end{array}$ & 19.2 & 30 & 92.4 & 96.4 & 0.024 \\
\hline SMAD7-W & TGF-beta signal pathway antagonist Smad7 & ChrUn & 4.47 & 4.95 & 93.8 & 93.6 & 0.208 \\
\hline SPIN-W & Spindlin & $\begin{array}{l}\text { ChrW } \\
\text { W_random }\end{array}$ & 1.8 & 10.6 & 95.7 & 98.5 & 0.045 \\
\hline STRSIA3-W & Sia-alpha-2,3-Gal-beta-1,4-GlcNAc-R: alpha 2,8-sialyltransferase-like & $\begin{array}{l}\text { ChrW } \\
\text { W_random }\end{array}$ & 4.31 & 3.16 & 94.7 & 95.7 & 0.105 \\
\hline SUB1-W & Activated RNA polymerase II transcriptional coactivator p15-like & ChrUn & 11.6 & 26.6 & 94.5 & 96.0 & 0.097 \\
\hline TXN-like1-W & thioredoxin & Chrun & 7.16 & 19.6 & 94.5 & 95.2 & 0.144 \\
\hline UBAP2-W & Ubiquitin associated protein 2 & $\begin{array}{l}\text { ChrW } \\
\text { W_random }\end{array}$ & 16.3 & 11.9 & 92.1 & 89.6 & 0.263 \\
\hline UBE2R2-W & Ubiquitin-conjugating enzyme E2R 2 & $\begin{array}{l}\text { ChrW } \\
\text { W_random }\end{array}$ & 5.38 & 17.4 & 94.4 & 100.0 & 0.000 \\
\hline VCP-like-W & Valosin containing protein & Chrun & 64.4 & 128 & 95.5 & 99.9 & 0.003 \\
\hline ZFR-W & Zinc finger RNA-binding protein & Autosome ChrUn & 4.47 & 7.51 & 93.8 & 94.1 & 0.172 \\
\hline ZNF532-W & Zinc finger protein 532-like & autosome & 3.52 & 5.59 & 94.0 & 94.0 & 0.181 \\
\hline
\end{tabular}


Table 1 Annotation and expression of chicken $\mathbf{W}$ chromosome genes. (Continued)

\begin{tabular}{|c|c|c|c|c|c|c|c|}
\hline ZSWIM6-W & Zinc finger SWIM domain-containing protein 6-like & $\begin{array}{l}\text { ChrW } \\
\text { W_random autosome ChrUn }\end{array}$ & 0.54 & 4.73 & 91.7 & 92.4 & 0.172 \\
\hline Mir-7b-W & MicroRNA 7b (in intron of HNRPK-W) & Chrun & unknown & unknown & 93.6 & & \\
\hline SNORD58-W 1 & Small nucleolar RNA SNORD58 (in intron of RPL17-W) & ChrUn & unknown & unknown & 97.0 & & \\
\hline SNORD58-W 2 & Small nucleolar RNA SNORD58 (in intron of RPL17-W) & Chrun & unknown & unknown & 89.7 & & \\
\hline SNORD121A-W 1 & Small nucleolar RNA SNORD121A (in intron of UBAP2-W) & $\begin{array}{l}\text { ChrW } \\
\text { W_random }\end{array}$ & unknown & unknown & 90.6 & & \\
\hline SNORD121A-W 2 & Small nucleolar RNA SNORD121A (in intron of UBAP2-W) & $\begin{array}{l}\text { ChrW } \\
\text { W_random }\end{array}$ & unknown & unknown & 90.4 & & \\
\hline
\end{tabular}

A list of the chicken W genes. Genomic Location gives the chromosome/s where the gene sequences can be found in the chicken reference genome (galGal4). Expression shows the average expression levels as an FPKM value (fragments per kilobase of exon per million fragments mapped) for females from the blastoderm and gonad (E4.5) tissue. The \% ID gives the percentage identity of the W gene's open read frame against its $Z$ gametologue for both DNA and amino acid sequences. dN/dS is the ratio of synonomous to non-synonomous substitutions, also across the open read frame of the W genes and their Z gametologues. 
fragments of the genome. To address this issue we performed a genome independent (de novo) transcript assembly using the ABySS software $[38,39]$ and used the de-novo assembled transcripts to reassemble different Cufflinks genes together. This enabled genes previously assigned to different regions of a chromosome or even across different chromosomes to be joined together into a single gene (see Additional file 1 for details). Figure 2 shows the results of such analysis for five representative genes, $R A S A-W$, ST8SIA-W, GOLPH-3-W, ZSWIM6-W and NEDD4-W (the remaining assembled $\mathrm{W}$ transcripts are given in Figure S4; see Additional file 1). Deduced complete transcripts are shown together with the sequences annotated by Ensembl and those derived from Cufflinks and ABySS assemblies. For example, the $R A S A-W$ transcript was assembled by joining seven sequences previously assigned partly to the $\mathrm{W}$ and partly to various fragments of the Un_random and W_random chromosome.

Using this approach, the open reading frames of Wlinked mRNAs could be completely assembled, with the exception of one gene, NEDD4-like-W. This resulted in the characterisation of ten genes where previously only part of their ORF was known, due to the poor assembly of the $\mathrm{W}$ chromosome. Of these genes, we estimate that, on average, $60 \%$ of open reading frame sequence was previously missing from that available in either Ensembl or on Genbank (Additional file 6). Subsequent expression analysis of $\mathrm{W}$ genes was carried out by mapping reads to the newly assembled complete cDNA sequences. Final FPKM values (Fragments Per Kilobase of exon per Million fragments mapped) are presented in Table 1.

The complete W-linked transcripts identified in this RNA-seq screen showed gene ontologies with varying functions, none of which are patently associated with sexual differentiation (Table 1 and Table S3 - Additional file 6). However, the lists included genes specifying proteins associated with transcriptional regulation (ZSWIM6W, ZNF532-W, MIER3-W, BTF3-W, SUB1-W), signalling (SMAD2-W, SMAD7-W, RASA1-W), the ubiquitination pathway (UBAP2-W, UBE2R2-W), the antioxidant thioredoxin (TXN-like-W), the ATP synthase, ATP5A1-W, and the aberrant nucleotide-binding protein, HINTW. As noted above, most W-linked genes did not show significant expression changes between the two tissues examined (Table 1). The most highly expressed genes across both tissues were HINT-W, RPL7-W, ATPA5A1-W, BTF3-W, $V C P$-like-W and hnRNKP.

\section{Confirmation of female-restricted expression and W- linkage}

The RNA-seq data demonstrated that the chicken W sex chromosome harbours more genes than previously thought and that these genes show robust transcriptional activity. All W genes were expressed in both blastoderms and E4.5 gonads. To validate the RNA-seq, quantitative RT-PCR was carried out on four representative genes, using W-gene specific primers. PCR amplification was detected in female but not male blastoderm and gonadal RNA samples (Figure 3A and 3B), thus confirming female specific expression. For some of these genes, whole mount in-situ hybridisation also confirmed female gonad-specific expression (Additional file 1, Figure S5). FISH mapping was used to validate $\mathrm{W}$ linkage, showing a single signal in female but not male cells (Figure 3B-E and Additional file 1, Figure S6). In addition, for predicted or novel genes not yet assigned to the $\mathrm{W}, \mathrm{PCR}$ analysis confirmed that they are present specifically in female but not male chicken genomic DNA (see Materials and Methods and Figure S6 in Additional file 1).

\section{Conservation and relative expression of W-linked genes and their $\mathrm{Z}$ gametologues}

The complete $\mathrm{W}$-linked transcripts assembled in this study were used to screen for homologues on the $\mathrm{Z}$ chromosome (gametologues). All W-linked genes with the exception of FAF (Female Associated Factor) were found to have gametologues on the $\mathrm{Z}$. There was high sequence homology between almost all W- and Z-linked copies at both the DNA level (average of $88.4 \%$ identity, Table 1) and the protein level (average of $90.3 \%$ identity, Table 1). An exception was the gene HINTW, which showed $41 \%$ sequence and $48.5 \%$ amino acid homology with its $\mathrm{Z}$ gametologue (Table 1). Evolution of novel function among $\mathrm{W}$-linked genes was examined by calculating the rates of synonymous and non-synonymous substitution for each Z-W gene pair [40] and is represented in Table 1, and as a sliding window across each gene pair in Figure S7 (Additional file 1). The dN/dS value for HINTW was the highest of all W genes (0.6) indicating that it has undergone the least amount of purifying selective pressure.

The combined expression of the $\mathrm{W}$ and $\mathrm{Z}$ gametologues was assessed in both blastoderms and gonads and is shown in Figure 4. For virtually all expressed Wlinked genes, the $\mathrm{Z}$ gametologue was also expressed, and in both tissues. (The exception was $F A F$, which lacks a $\mathrm{Z}$ gametologue). Total expression from the $\mathrm{W}$ and $\mathrm{Z}$ gametologues in females was in most cases comparable to the expression of the two Z-linked copies in males, where typically the $\mathrm{Z}$ and $\mathrm{W}$ contributed equally to the total expression in females (Figure 4A, B). This suggests that most $\mathrm{W} / \mathrm{Z}$ gametologues in the chicken embryo effectively operate in an autosomal-like fashion (having two functional copies in both male and female). However in some cases, the combined $\mathrm{W} / \mathrm{Z}$ gene expression in females was significantly higher than the total Zlinked expression in males and was primarily due to $\mathrm{W}$ transcription. In the blastoderm, this was the case for 








Figure 3 Validation of RNA-seq by quantitative RT-PCR and Confirmation of W-linkage. (A) Blastoderm expression analysis of four representative W genes, KCMF-W, RASA-W, MIER3-W and ZNF532. Expression was detectable in females (red) but not in males (blue). Normalised W gene expression is shown; mean $+/-$ SEM; $n=3$; ${ }^{* *} P<0.05$. (B-E) FISH mapping of genes identified by RNA-seq to the W sex chromosome in female chicken metaphase spreads. BAC clones were used as probes. (B) BAC clone Ch261-113E6 (ZNR-W, BTF3-W) (red) and BAC Ch261-178N8 (RASA1-W, BTF3-W) (green). (C) BAC clone Ch261-107E4 (HNRPK2-W, GOLPH3-W (red). (D) BAC clone Ch261-60P24 (ZNF532-W, SnoR58-W) (red). (E) BAC clone Ch261-114G22 (UBE2R2-W, RASA1-W, SnOR121A-W) (red). Metaphase chromosomes are stained with DAPI (blue). A single signal was detected in each case and only in female cells, confirming $W$ linkage. 





HINT, SMAD2 and MIER-3(Figure 4A). In E4.5 gonads, female expression was higher for HINT, MIER3, the putative transcription factor ZSWIM6, VCP-like (Valosin-containing protein) and ST8SIA3 (a sialyltransferaselike gene) (Figure 4B).

\section{Discussion}

This study indicates that cells of the chicken embryo commence sexual differentiation at the molecular level early in development, through sexually dimorphic gene expression from both sex chromosomes and autosomes. This involves the enigmatic W sex chromosome, and it supports recent observations indicating that sexual differentiation may be at least partly cell autonomous in birds. This does not, however, exclude an important role for non-cell autonomous sexual differentiation, which involves hormonal signalling and is clearly very important in birds as it is in all animals. However, the data presented here support the notion that cell autonomous factors are also important, and there is very likely an interaction between both mechanisms to ensure complete and proper phenotypic sexual differentiation.

Previous studies have also reported sexually dimorphic gene expression in chicken embryos pre-dating gonadal development [32,41,42]. These studies utilised microarrays or subtractive hybridisation to uncover genes regulated in a sex-specific manner prior to gonadal differentiation in the chicken embryo. Our study utilised the earliest time point to date ( $12 \mathrm{~h}$, stage 1 blastoderms), and has employed the most sensitive and comprehensive method available to assay gene expression. RNA-seq detects all expressed genes, including those that are unannotated, compared to microarray analysis, which only detects sequences arrayed on the microarray [29]. The finding that sexually dimorphic gene expression can pre-date gonadal sex differentiation is not limited to birds, but is likely to be a feature of all animals. Sex differences in gene expression before gonadogenesis have been reported in mouse and bovine embryos [43,44].

We found that W- and Z-linked genes showing sexually dimorphic expression in blastoderms largely maintained this differential expression profile in the gonads, pointing to a pervasive role for the sex chromosomes in the continuity of cell autonomous sexual development. The sexually dimorphic expression of autosomal genes indicates that female- and male-specific pathways are engaged as early as the blastoderm stage. However, a different set of autosomal genes showed sexually dimorphic expression in the undifferentiated gonads, most notably genes implicated in gonadal development. This indicates that the sex chromosomes activate different sex-specific pathways in different tissues.

The mechanism through which the $\mathrm{Z}$ and $\mathrm{W}$ sex chromosomes act to direct sexual differentiation pathways in early embryos is still unclear. One hypothesis is that the W chromosome carries a dominant-acting female determinant. This could confer 'femaleness' in a cell autonomous fashion early in development, but it may also initiate ovarian differentiation of the gonads at day 4.5. The RNA-seq data presented here has allowed us to draw some conclusions regarding the potential role of the $\mathrm{W}$ in avian sex determination. Contrary to previous assumptions, we found that the $\mathrm{W}$ is highly transcriptionally active in chicken embryos. Our novel analysis strategy allowed us to construct full-length transcripts for essentially the entire $\mathrm{W}$ transcriptome in two tissues. Using these we could correct and expand the annotation of the chicken W, providing complete transcript sequences and open reading frames for almost all 26 genes (Table 1 and Table S3 - Additional file 6). Most of these genes have previously been reported as W-linked in chicken $[20,22]$. However, a thorough assessment of expression prior to or during gonadal development has been lacking, and limited sequence analysis has been carried out for these W-linked genes. In addition, we describe here two additional W linked genes and three W-linked small RNAs (Table 1). The assembly of full-length $W$ transcripts described has allowed the accurate comparison of $\mathrm{W}$ and $\mathrm{Z}$ gametologues, providing key information on the degree of divergence between gene pairs and their relative expression levels. Most of the expressed W genes have open reading frames that are almost or completely homologous to their Z-linked gametologues (Additional file 1, Figure S7), which were also expressed in both blastoderms and gonads. This includes both the known and novel genes identified here. These $\mathrm{W}$ and $\mathrm{Z}$ genes are therefore likely to be acting as 'de facto autosomal' pairs, and hence almost all W-linked genes are unlikely female sex determinants. Indeed most of these genes encode proteins associated with general functions in the cell, such as ubiquitination, lipid and energy metabolism, and chromosome structure (Table 1). The two exceptions are HINTW, which is divergent from its $\mathrm{Z}$ gametologue, and $F A F$, which lacks a $\mathrm{Z}$ gametologue.

HINTW ( Histidine Triad nucleotide binding, Wlinked) is highly expressed in female chicken embryos at the blastoderm and gonadal stages (Table 1) [17]. The high level of HINTW expression in females most likely reflects the multiple copies of HINTW that reside on the chicken W [17]. While an endogenous HINTW protein has yet to be demonstrated, it is predicted to specify an aberrant nucleotide binding protein, lacking a key catalytic domain. The Z-linked copy, HINTZ, encodes a bona fide nucleotide binding protein, mediating the hydrolysis of nucleoside monophosphates [17] and it is hypothesised that HINTW may operate in a dominant negative fashion, blocking the function of HINTZ $[8,45]$. While experimental over-expression of HINTW mRNA 
fails to alter male development in chicken embryos [46], the potential role of HINTW in avian sex determination requires closer examination. One $\mathrm{W}$-linked transcript detected in our screen did not have a $\mathrm{Z}$ gametologue; FAF (Female Associated Factor) [23]. The FAF gene product shows no clear homology to known proteins and its potential role in sexual differentiation is not clear at this stage.

The same set of W-linked genes expressed in female blastoderms was also expressed in E4.5 female gonads, yet a different set of autosomal genes was expressed. Therefore, if W-linked genes play a role in female development, they must engage different pathways in the different tissues. In the developing female gonad, a putative ovary determinant would directly or indirectly activate expression of the key FOXL2 gene, which is thought to then activate CYP19A1 and hence the oestrogen synthesis that is central to ovarian differentiation. Since we detected female enriched FOXL2 expression already in E4.5 gonads, the direct activator of this gene must also be present in our E4.5 female dataset (Additional file 5). This requires further analysis.

The alternative mechanism mediating avian sex determination involves $\mathrm{Z}$-gene dosage. As there is no global $\mathrm{Z}$ chromosome dosage compensation system in birds, a sex determining mechanism may rely upon $\mathrm{Z}$ gene dosage inequality between the sexes. One or more non-dosage compensated Z-linked genes could direct sexual differentiation in individual cells. Our findings indicate that like the W, many Z-linked genes show sexually dimorphic expression that does not differ between the tissues assayed, with only $1 \%$ showing significantly different levels of dimorphic expression between the two tissues. This indicates that the sex chromosome influence sexual differentiation in cells, but this does not explain how sex-linked genes could then trigger sexually dimorphic expression of different autosomal genes in each tissue. Many Z- and Wlinked genes showed different average expression levels between tissues (Figure 1D), and therefore while female: male ratios may not differ, changes in total expression levels may play a role in each tissue. For example, the Z-linked testis-associated genes, DMRT1 and HEMOGEN, are more highly expressed in the gonads compared to the blastoderms. Therefore it is possible that additional Z-linked genes may play a role in sexual differentiation in different tissues through changes in their relative expression. It is noteworthy in this regard that many Z-linked genes with sexually dimorphic expression in the gonads were sex-related (Additional file 5). Previous studies have reported a clear bias for genes associated with sex and reproduction located on the avian $\mathrm{Z}$ sex chromosome $[47,48]$, making sex determination via $\mathrm{Z}$ dosage and expression levels an attractive model.
It has recently been hypothesised that multiple parallel pathways leading to sexual differentiation probably exist in mammalian embryos [49]. Similarly, in birds, molecular pathways controlling sexual differentiation in a cell autonomous fashion in the early embryo would be different to those operating specifically to induce ovarian versus testicular development at later stages. This idea is supported by our findings that different sets of autosomal genes show sexually dimorphic expression in the blastoderm compared to the E4.5 embryonic gonad. It is noted, however, that ultimately, proper sexual differentiation must involve the interaction of both cell autonomous and non-cell autonomous factors. Despite the advances brought by this research, the exact mechanism underlying avian sex determination is still unclear.

\section{Conclusions}

The data reported here show sexually dimorphic gene expression in chicken blastoderms and gonads prior to gonadal sex differentiation. This supports the notion that sexual differentiation begins at the molecular level in a cell autonomous manner in the chicken embryo. This involves both sex-linked and autosomal genes, with different sets of genes being expressed in the two tissues, demonstrating the engagement of different sexually dimorphic pathways. While sexually dimorphic gene expression from the $\mathrm{Z}$ and $\mathrm{W}$ sex chromosomes does not dramatically differ between developmental stages, we show that significant differences in average levels of expression of sex-linked genes may be responsible for activation of different signalling pathways in a tissue specific manner. Furthermore, we comprehensively define the $\mathrm{W}$ chromosome transcriptome for two early embryonic tissues, annotating $\mathrm{W}$ genes and providing complete open reading frames for most genes, and characterising two novel W-linked genes and several small RNAs.

\section{Materials and methods \\ Tissue collection}

All experiments were carried out on a single line of Specific Pathogen Free (SPF) embryos (SPAFAS) from the SPF White Leghorn strain of chick (Lohman-LSL).

Blastoderms: Eggs were incubated for $12 \mathrm{~h}$ at $38^{\circ} \mathrm{C}$ and the blastoderms were dissected in cold DEPC-treated PBS. Hamburger and Hamilton (HH) stage 1 blastoderms were taken, and any showing developed primitive streaks were discarded. Only the area pellucida was dissected from the vitelline membrane and used, and any remaining yolk was removed. A small piece of blastoderm was taken for genetic sexing by PCR (see below), and the remainder of the blastoderm was stored at $-80^{\circ} \mathrm{C}$ until RNA extraction was performed. 
Gonads: SPF eggs were incubated until stage 26 (embryonic day 4.5). Paired gonads were removed and stored at $-80^{\circ} \mathrm{C}$. Handplate tissue was used for sexing. Embryos were sexed by PCR as described previously $[50,51]$.

\section{RNA extraction and sequencing}

Tissues were pooled according to sex. Twelve blastoderms or 16 paired gonads were pooled for each replicate (two male replicates and two female replicates were collected for each tissue). Total RNA was extracted using the RNeasy micro kit (QIAGEN) (which enriches for mRNAs, that is, RNAs $>200$ base pairs in length). On column DNAsing was performed to remove contaminating genomic DNA. The resulting RNA was poly A-selected, reverse transcribed, fragmented, bar-coded and sequenced using the Illumina HiSeq2000 at Australian Genome Research Facility (AGRF) in Melbourne. We sequenced 100 base pair, paired ends reads. Four lanes were used and all samples were bar-coded and run on each lane. The RNA was sequenced to a depth of approximately 20 million readpairs per sample per lane, giving a total for each sample of 80 million read-pairs (160 million reads). These data are available on the NCBI Sequence Read Archive (accession number SRA055442).

\section{RNA-seq analysis}

Sequences were screened to remove poor quality bases, and subsequent analysis of the data followed the three methods shown in Additional file 1, Figure S8 and described in detail in the Supplementary Methods (Additional file 1). Briefly, we mapped the trimmed reads to the genome and counted reads which overlapped Ensembl genes, we performed genome-guided transcriptome discovery using Cufflinks [52] and we assembled the transcriptome independent of the chicken genome using ABySS [38][39]. For the Ensembl differential expression analysis and W-linked gene identification we mapped the read-pairs to the chicken genome, galGal3, using the TopHat 1.3.1 software [26], with default settings. We counted reads which mapped to annotated genes giving a count for each gene in each sample and then used edgeR for differential expression (DE) analysis. edgeR uses a Generalized Linear Modeling (GLM) framework to model the gene counts as a negative binomial distribution. Variation estimates are stabilized by borrowing information between genes using an empirical Bayes approach [27] and hypothesis tests are performed using a likelihood ratio test. This method takes into account both biological and technical sources of variation even for small numbers of replicates. In this case we had 3 degrees of freedom to estimate biological variability [53]. Further details are described in the Sup- plementary Methods (Additional file 1). Differential expression for the Cufflinks defined genes was performed in a similar manner to the Ensembl analysis, however we mapped to the updated chicken genome Galgal4 and the set of chicken transcripts was created by running Cufflinks 1.3.0 on the mapped reads (see Additional file 1 - Supplementary Methods).

\section{De-novo $W$ gene assembly}

The de-novo transcriptome assembly was performed using Abyss version 1.3.2 on reads from all samples [39]. To obtain the full sequence of genes split over non-contiguous genomic regions, we merged the Ensembl, Cufflinks and Abyss W transcripts using CAP3 [54]. This was achieved using the novel approach of scaffolding the transcripts using the sequence of their $\mathrm{Z}$ gametologues, as detailed in the Supplementary Methods (Additional file 1). Our denovo assembled $\mathrm{W}$ gene sequences and their $\mathrm{Z}$ gametologue sequences are provided as Additional files 7 and 8.

\section{Preparation of chromosomes and fluorescent in-situ hybridization (FISH) of BAC clones}

Mitotic metaphase chromosomes and interphase preparations were generated from the established chicken embryonic fibroblast cell lines. Fluorescent in-situ hybridization was carried out as described in the Supplementary Methods (Additional file 1).

\section{Quantitative reverse transcription-PCR (qRT-PCR)}

Dissected stage 1-4 blastoderms were pooled according to sex, including three biological replicates, RNA was extracted and reverse transcribed as previously described [55]. Quantitative real-time PCR was carried out using the Roche UPL Assay Design Center and relative expression was determined using the comparative $\mathrm{CT}$ method $(\triangle \Delta \mathrm{CT})$, with samples normalized against HPRT and expressed as fold change, as described in the Supplementary methods (Additional file 1).

\section{Additional material}

Additional file 1: Supplementary figures (1 to 10), supplementary methods, figure legends for supplementary figures and tables.

Additional file 2: Table S1a. Dimorphically expressed Ensembl annotated genes in the Blastoderm.

Additional file 3: Table S1b. Dimorphically expressed Ensembl annotated genes in the E4.5 gonads.

Additional file 4: Table S1c. Two tests for dimorphic expression.

Additional file 5: Table S2. Genes previously implicated in sexual reproduction or gonadogenesis in a variety of models.

Additional file 6: Table S3. Ensembl Identifiers for W-linked genes and their Z gametologues.

Additional file 7: W gene de-novo assembly sequences (fasta file). Additional file 8: Z gene de-novo assembly sequences (fasta file). 


\section{Abbreviations}

DE: differential expression; FDR: false discovery rate; FISH: fluorescent in situ hybridization; FPKM: fragments per kilobase of exon per million fragments mapped; GLM: generalised linear modelling framework; $\mathrm{HH}$ : Hamburger and Hamilton; MHM: male hypermethylated; qRT-PCR: quantitative reverse transcription-PCR; RNA-seq: next-generation sequencing of RNA; SPF: specific pathogen free.

\section{Authors' contributions}

$\mathrm{KA}, \mathrm{AO}, \mathrm{CS}$ and $\mathrm{AS}$ designed the RNA seq screen. KA and CS dissected and extracted RNA. ND carried out bioinformatics analysis under guidance of AO. KA carried out lab validation and confirmation of RNA seq results and helped interpret data. KR assisted with lab work. DD and FG carried out FISH analysis. KA, CS, ND and AS wrote the manuscript. All authors read and approved the final manuscript.

\section{Competing interests}

The authors declare that they have no competing interests.

\section{Acknowledgements}

We thank the Australian Genomic Research Institute (AGRF) in Melbourne. AO is supported by NHMRC Career Development Fellowship (1051481). KA is supported by the Poultry CRC for Sex Determination. CS is funded through an Australian Research Council (ARC) Future Fellowship, while KR is funded through an ARC Discovery Project Grant. AS is supported by an NHMRC Program grant (546517). FG is an ARC Research Fellow. DD is supported by a PhD scholarship from the Malaysian Government. This research was conducted within the Poultry CRC, established and supported under the Australian Government's Cooperative Research Centres Program. MCRI is supported by the Victorian Government's Operational Infrastructure Support Program.

\section{Author details}

'Murdoch Childrens Research Institute, Royal Childrens Hospital, Flemington Road, Parkville 3054 Melbourne, VIC, Australia. ${ }^{2}$ Poultry Cooperative Research Centre, Armidale, NSW, Australia. ${ }^{3}$ Department of Genetics, The University of Melbourne, Parkville, 3054, Melbourne, VIC, Australia. ${ }^{4}$ Institute of Biological Sciences, Faculty of Science, University of Malaya, 50603, Kuala Lumpur, Malaysia. ${ }^{5}$ The Robinson Institute, School of Molecular and Biomedical Science, University of Adelaide, 5005 Adelaide, SA, Australia. ${ }^{6}$ Department of Paediatrics, The University of Melbourne, Flemington Road, Parkville, 3054 Melbourne, VIC, Australia.

Received: 23 November 2012 Revised: 14 March 2013

Accepted: 25 March 2013 Published: 25 March 2013

\section{References}

1. Ferguson-Smith M: The evolution of sex chromosomes and sex determination in vertebrates and the key role of DMRT1. Sex Dev 2007, 1:2-11.

2. Griffin DK: Is the $Y$ chromosome disappearing?-both sides of the argument. Chromosome Res 2012, 20:35-45.

3. McBride D, Sang H, Clinton M: Expression of Sry-related genes in the developing genital ridge/mesonephros of the chick embryo. J Reprod Fertil 1997, 109:59-63.

4. Nanda I, Benisch P, Fetting D, Haaf T, Schmid M: Synteny conservation of chicken macrochromosomes 1-10 in different avian lineages revealed by cross-species chromosome painting. Cytogenet Genome Res 2008, 132:165-181.

5. Bellott DW, Skaletsky H, Pyntikova T, Mardis ER, Graves T, Kremitzki C, Brown LG, Rozen S, Warren WC, Wilson RK, Page DC: Convergent evolution of chicken $\mathrm{Z}$ and human $\mathrm{X}$ chromosomes by expansion and gene acquisition. Nature 2010, 466:612-616.

6. Fridolfsson AK, Cheng H, Copeland NG, Jenkins NA, Liu HC, Raudsepp T, Woodage T, Chowdhary B, Halverson J, Ellegren H: Evolution of the avian sex chromosomes from an ancestral pair of autosomes. Proc Natl Acad Sci USA 1998, 95:8147-8152.

7. Ellegren $\mathrm{H}$ : Evolution of the avian sex chromosomes and their role in sex determination. Trends Ecol Evol 2000, 15:188-192.

8. Smith CA: Sex determination in birds: HINTs from the W sex chromosome?. Sex Dev 2007, 1:279-285.
9. Smith CA, Roeszler KN, Ohnesorg T, Cummins DM, Farlie PG, Doran TJ, Sinclair AH: The avian Z-linked gene DMRT1 is required for male sex determination in the chicken. Nature 2009, 461:267-271.

10. Nakata T, Ishiguro M, Aduma N, Izumi H, Kuroiwa A: Chicken hemogen homolog is involved in the chicken-specific sex-determining mechanism. Proc Natl Acad Sci USA 2013, doi: 10.1073/pnas.1218714110.

11. Saitoh Y, Mizuno S: Distribution of Xhol and EcoRI family repetitive DNA sequences into separate domains in the chicken W chromosome. Chromosoma 1992, 101:474-477.

12. Itoh Y, Mizuno S: Molecular and cytological characterization of Ssplfamily repetitive sequence on the chicken W chromosome. Chromosome Res 2002, 10:499-511.

13. Consortium ICG: Sequence and comparative analysis of the chicken genome provide unique perspectives on vertebrate evolution. Nature 2004, 432:695-716.

14. Chen N, Bellott DW, Page DC, Clark AG: Identification of avian W-linked contigs by short-read sequencing. BMC Genomics 2012, 13:183.

15. Consortium ICG: Gallus gallus 4.0 2011, 22/11/2011.

16. Ellegren $\mathrm{H}$ : First gene on the avian $\mathrm{W}$ chromosome (CHD) provides a tag for universal sexing of non-ratite birds. Proc Biol Sci 1996, 263:1635-1641.

17. Hori T, Asakawa S, Itoh Y, Shimizu N, Mizuno S: Wpkci, encoding an altered form of $\mathrm{PKCl}$, is conserved widely on the avian W chromosome and expressed in early female embryos: implication of its role in female sex determination. Mol Biol Cell 2000, 11:3645-3660.

18. Axelsson E, Smith NG, Sundstrom H, Berlin S, Ellegren H: Male-biased mutation rate and divergence in autosomal, $z$-linked and $w$-linked introns of chicken and Turkey. Mol Biol Evol 2004, 21:1538-1547.

19. Wahlberg P, Stromstedt L, Tordoir X, Foglio M, Heath S, Lechner D, Hellstrom AR, Tixier-Boichard M, Lathrop M, Gut IG, Andersson L: A highresolution linkage map for the $Z$ chromosome in chicken reveals hot spots for recombination. Cytogenet Genome Res 2007, 117:22-29.

20. Nam K, Ellegren H: The chicken (Gallus gallus) Z chromosome contains at least three nonlinear evolutionary strata. Genetics 2008, 180:1131-1136.

21. Ensembl: Chicken genome Release 67

22. Moghadam HK, Pointer MA, Wright AE, Berlin S, Mank JE: W chromosome expression responds to female-specific selection. Proc Natl Acad Sci USA 2012, doi: 10.1073/pnas.1202721109.

23. Zhao D, McBride D, Nandi S, McQueen HA, McGrew MJ, Hocking PM, Lewis PD, Sang HM, Clinton M: Somatic sex identity is cell autonomous in the chicken. Nature 2010, 464:237-242.

24. Clinton M, Zhao D, Nandi S, McBride D: Evidence for avian cell autonomous sex identity (CASI) and implications for the sexdetermination process?. Chromosome Res 2012, 20:177-190.

25. Hamburger $\mathrm{V}$, Hamilton $\mathrm{HL}$ : A series of normal stages in the development of the chick embryo. J Morph 1951, 88:49-92.

26. Trapnell C, Pachter L, Salzberg SL: TopHat: discovering splice junctions with RNA-Seq. Bioinformatics 2009, 25:1105-1111.

27. Robinson MD, McCarthy DJ, Smyth GK: edgeR: a Bioconductor package for differential expression analysis of digital gene expression data. Bioinformatics 2010, 26:139-140.

28. Smith CA, McClive PJ, Hudson QJ, Sinclair AH: Male-specific cell migration into the developing gonad is conserved between mammals and birds and involves PDGF signalling. Developmental Biology 2005, 284:337-350.

29. Oshlack A, Robinson MD, Young MD: From RNA-seq reads to differential expression results. Genome Biol 2010, 11:220.

30. Itoh Y, Melamed E, Yang X, Kampf K, Wang S, Yehya N, Van Nas A, Replogle K, Band MR, Clayton DF, Schadt EE, Lusis AJ, Arnold AP: Dosage compensation is less effective in birds than in mammals. J Biol 2007, 6:2.

31. Arnold AP, Itoh Y, Melamed E: A bird's-eye view of sex chromosome dosage compensation. Annu Rev Genomics Hum Genet 2008, 9:109-127.

32. Zhang SO, Mathur S, Hattem G, Tassy O, Pourquie O: Sex-dimorphic gene expression and ineffective dosage compensation of Z-linked genes in gastrulating chicken embryos. BMC Genomics 2010, 11:13.

33. Itoh Y, Kampf K, Arnold AP: Possible differences in the two $Z$ chromosomes in male chickens and evolution of MHM sequences in Galliformes. Chromosoma 2011, 120:587-598.

34. Teranishi M, Shimada Y, Hori T, Nakabayashi O, Kikuchi T, Macleod T, Pym R, Sheldon B, Solovei I, Macgregor H, Mizuno S: Transcripts of the MHM region on the chicken $Z$ chromosome accumulate as non-coding RNA in the nucleus of female cells adjcent to the DMRT1 locus. Chromosome Res 2001, 9:147-165. 
35. Dennis G Jr, Sherman BT, Hosack DA, Yang J, Gao W, Lane HC, Lempicki RA: DAVID: Database for Annotation, Visualization, and Integrated Discovery. Genome Biol 2003, 4:P3.

36. Waxman DJ, O'Connor C: Growth hormone regulation of sex-dependent liver gene expression. Mol Endocrinol 2006, 20:2613-2629.

37. Trapnell C, Williams BA, Pertea G, Mortazavi A, Kwan G, van Baren MJ, Salzberg SL, Wold BJ, Pachter L: Transcript assembly and quantification by RNA-Seq reveals unannotated transcripts and isoform switching during cell differentiation. Nat Biotechnol 2010, 28:511-515.

38. Simpson JT, Wong K, Jackman SD, Schein JE, Jones SJ, Birol I: ABySS: a parallel assembler for short read sequence data. Genome Res 2009, 19:1117-1123.

39. Robertson G, Schein J, Chiu R, Corbett R, Field M, Jackman SD, Mungall K, Lee S, Okada HM, Qian JQ, Griffith M, Raymond A, Thiessen N, Cezard T, Butterfield YS, Newsome R, Chan SK, She R, Varhol R, Kamoh B, Prabhu AL, Tam A, Zhao Y, Moore RA, Hirst M, Marra MA, Jones SJ, Hoodless PA, Birol I: De novo assembly and analysis of RNA-seq data. Nat Methods 2010, 7:909-912

40. Charif D, Thioulouse J, Lobry JR, Perriere G: Online synonymous codon usage analyses with the ade 4 and seqinR packages. Bioinformatics 2005 , 21:545-547.

41. Yamada D, Koyama $Y$, Komatsubara M, Urabe M, Mori M, Hashimoto $Y$, Nii R, Kobayashi M, Nakamoto A, Ogihara J, Kato J, Mizuno S: Comprehensive search for chicken W chromosome-linked genes expressed in early female embryos from the female-minus-male subtracted cDNA macroarray. Chromosome Res 2004, 12:741-754.

42. Lin YP, Chen LR, Chen CF, Liou JF, Chen YL, Yang JR, Shiue YL: Identification of early transcripts related to male development in chicken embryos. Theriogenology 2010, 74:1161-1178, e1161-1168.

43. Nef S, Schaad O, Stallings NR, Cederroth CR, Pitetti JL, Schaer G, Malki S, Dubois-Dauphin M, Boizet-Bonhoure B, Descombes P, Parker KL, Vassalli JD: Gene expression during sex determination reveals a robust female genetic program at the onset of ovarian development. Dev Biol 2005, 287:361-377.

44. Bermejo-Alvarez P, Rizos D, Rath D, Lonergan P, Gutierrez-Adan A: Sex determines the expression level of one third of the actively expressed genes in bovine blastocysts. Proc Natl Acad Sci USA 2010, 107:3394-3399.

45. Moriyama S, Ogihara J, Kato J, Hori T, Mizuno S: PKCI-W forms a heterodimer with PKCl-Z and inhibits the biological activities of PKCl-Z in vitro, supporting the predicted role of PKCI-W in sex determination in birds. J Biochem 2006, 139:91-97.

46. Smith CA, Roeszler KN, Sinclair AH: Genetic evidence against a role for W-linked histidine triad nucleotide binding protein (HINTW) in avian sex determination. Int J Dev Biol 2009, 53:59-67.

47. Storchova R, Divina P: Nonrandom representation of sex-biased genes on chicken Z chromosome. J Mol Evol 2006, 63:676-681.

48. Naurin $S$, Hasselquist $D$, Bensch $S$, Hansson B: Sex-biased gene expression on the avian $\mathrm{z}$ chromosome: highly expressed genes show higher malebiased expression. PLoS One 2012, 7:e46854.

49. Arnold AP: The end of gonad-centric sex determination in mammals. Trends Genet 2011, 28:55-61.

50. Kodama H, Saitoh H, Tone M, Kuhara S, Sakaki Y, Mizuno S: Nucleotide sequences and unusual electrophoretic behavior of the W chromosomespecific repeating DNA units of the domestic fowl, Gallus gallus domesticus. Chromosoma 1987, 96:18-25.

51. Clinton M, Haines L, Belloir B, McBride D: Sexing chick embryos: a rapid and simple protocol. Br Poult Sci 2001, 42:134-138.

52. Trapnell C, Roberts A, Goff L, Pertea G, Kim D, Kelley DR, Pimentel H, Salzberg SL, Rinn JL, Pachter L: Differential gene and transcript expression analysis of RNA-seq experiments with TopHat and Cufflinks. Nat Protoc 2012, 7:562-578.

53. McCarthy DJ, Chen Y, Smyth GK: Differential expression analysis of multifactor RNA-Seq experiments with respect to biological variation. Nucleic Acids Res 2012, 40:4288-4297.

54. Huang X, Madan A: CAP3: A DNA sequence assembly program. Genome Res 1999, 9:868-877.

55. Smith CA, Shoemaker CM, Roeszler KN, Queen J, Crews D, Sinclair AH: Cloning and expression of R-Spondin1 in different vertebrates suggests a conserved role in ovarian development. BMC Dev Biol 2008, 8:72. doi:10.1186/gb-2013-14-3-r26

Cite this article as: Ayers et al:: RNA sequencing reveals sexually dimorphic gene expression before gonadal differentiation in chicken and allows comprehensive annotation of the W-chromosome. Genome Biology 2013 14:R26.

\section{Submit your next manuscript to BioMed Central and take full advantage of:}

- Convenient online submission

- Thorough peer review

- No space constraints or color figure charges

- Immediate publication on acceptance

- Inclusion in PubMed, CAS, Scopus and Google Scholar

- Research which is freely available for redistribution

Submit your manuscript at www.biomedcentral.com/submit
Biomed Central 